\title{
Clinical frailty score as an independent predictor of outcome in COVID-19 hospitalised patients
}

\author{
Gouri Koduri ${ }^{2}$ - Sriya Gokaraju ${ }^{1} \cdot$ Maria Darda $^{1} \cdot$ Vinod Warrier $^{3} \cdot$ Irina Duta $^{3} \cdot$ Fiona Hayes $^{2} \cdot$ Iman El Sayed $^{4}$. \\ Yasser Noeman-Ahmed ${ }^{1,5,6}$
}

Received: 16 October 2020 / Accepted: 29 April 2021 / Published online: 4 June 2021

(c) European Geriatric Medicine Society 2021

\section{Key summary points}

Aim To explore potential predictive variables associated with outcomes using baseline clinical parameters in 500 hospitalised COVID-19 patients.

Findings Older age, clinical frailty score and C-reactive protein are independent predictors of mortality.

Message Integrated frailty and age-based risk stratification are essential to allow for early intervention to improve patient outcomes.

\begin{abstract}
Purpose of the study We explored potential predictive variables associated with outcomes using baseline clinical parameters of 500 hospitalised patients with COVID -19 in a single centre, UK.

Methods Retrospective study collecting demographic and clinical characteristics of patients admitted at Southend University Hospital from 20th February to 7th May 2020.

Results The mean age of the cohort admitted to hospital with Covid-19 was 69.4 and 58\% were over 70. Comorbidities were more frequently observed in non-survivors, whose mean Clinical Frailty Scale was significantly higher (5 vs 3 ) than survivors, $p<0.001$. In addition, mean C-reactive protein was significantly higher.

Conclusion Older and frailer patients with high inflammatory markers were at risk of poor outcomes. Integrated frailty and age-based risk stratification is essential, in addition to monitoring saturation /FiO2 ratio (SFR) and inflammatory markers throughout the disease course to allow for early intervention to improve patient outcomes. A frailty-based risk-stratification approach, rather than age may prove more valuable when considering interventions in patients with multiple comorbidities.
\end{abstract}

Keywords COVID-19 · Epidemiological characteristics $\cdot$ Sars-Cov-2 $\cdot$ Prognostic factors

Yasser Noeman-Ahmed

Yasser.ahmed1@nhs.net

1 Respiratory Department, Mid and South Essex NHS Foundation Trust, Southend University Hospital, Westcliff-on-Sea, Essex, UK

2 Rheumatology Department, Mid and South Essex NHS Foundation Trust, Southend University Hospital, Westcliff-on-Sea, Essex, UK

3 Department of Medicine, Mid and South Essex NHS Foundation Trust, Southend University Hospital, Westcliff-on-Sea, Essex, UK

4 Department of Biomedical Informatics and Medical Statistics, Medical Research Institute, Alexandria University, Alexandria, Egypt

5 Critical Care Medicine Department, Faculty of Medicine, Alexandria University, Alexandria, Egypt

6 Department of Respiratory Medicine, Lecturer Critical Care Medicine, Southend University Hospital, Alexandria University, Alexandria, Egypt 


\section{Introduction}

The coronavirus disease 19 (COVID-19) pandemic is one of the worst infectious disease outbreaks of recent times; with the first wave in the UK, we have encountered 312,000 confirmed cases and 44,819 fatalities [1]. COVID-19 is characterized by a highly variable course. While most patients experience only mild symptoms, a relevant proportion of patients develop severe disease progression up to respiratory failure. Several factors and mechanisms are proposed to influence COVID-19 pathogenesis. The most notable risk factor is age, followed by co-morbidities, including diabetes, obesity, cardiovascular and cerebrovascular diseases [2-5].

The mortality rate is variable; this is because of differences in the population demographics, the method used to register COVID-19, and the health services [6]. A recent report showed that mortality rate was $5.6 \%$ for China and $15.2 \%$ outside of China [7]. Belgium has relatively high case fatality rates $(16.34 \%)$, followed by France $(15.65 \%)$, UK (14.21\%), Italy (14.15\%), Hungary (13.07\%), Netherlands $(12.91 \%)$, Sweden $(12.21 \%)$ and USA $(5.95 \%)$. The mortality excess has been primarily seen in the age group of $\geq 65$ years globally with higher case fatality rates in older patients with comorbidities [8,9]

In most studies, older age and co-morbidities have consistently shown to be associated with poor outcomes and aging process is known to increase frailty. In addition, there is substantial evidence that frailty is associated with worse outcomes in both medical and surgical patients, prolonged length of stay, increased care needs on discharge and mortality $[10,11]$. Thus, it is very likely that frailty, together with comorbidities, may have contributed to the high mortality from COVID-19 among older people. Only few studies have evaluated Clinical Frailty Score (CFS) in COVID -19 [12, 13].

Therefore, our specific aim was to assess if CFS, was an independent predictor which has not been extensively described in existing literature as an efficient tool for assessing frailty, since this may be significant in determining outcomes for older patients. We hope that by adding to this growing body of evidence, we can assist early intervention in these patients to prevent rapid clinical deterioration and offer medications that have shown evidence in improving outcomes [14, 15].

\section{Methodology}

In this retrospective study, 500 patients with proven RTPCR assay of nasopharyngeal swab positive and/or high likelihood of SARS COVID-19 infection with clinical and radiographic evidence who were admitted to Southend
University Hospital from 20th February to 7th May 2020 were enrolled.

Collected demographic and disease characteristics included age, sex, ethnicity, clinical signs and symptoms at presentation and baseline observations. Laboratory findings included full blood count, neutrophil to lymphocyte ratio (NLR), C-reactive protein (CRP) and renal function, as well as ferritin, D-dimer, lactate dehydrogenase (LDH) and troponin, where available.

Patients' oxygen saturations $\left(\mathrm{SpO}_{2}\right)$ and supplementary oxygen $\left(\mathrm{FiO}_{2}\right)$ were recorded. $\mathrm{SpO}_{2}$ to $\mathrm{FiO}_{2}$ ratio (SFR) was calculated. The SFR has previously been shown to have a promising role to predict ITU admission [16]. Rice et al. were able to describe a relation between $\mathrm{PaO} 2 / \mathrm{FiO} 2$ and SFR. An SFR of 235 and 315 corresponded to $\mathrm{PaO} 2 / \mathrm{FiO} 2$ of 200 and 300 with a sensitivity of $85 \%$ and $91 \%$; and specificity of $85 \%$ and $91 \%$, respectively [17]. Bilan et al. also were able to demonstrate the reliability of SFR for the diagnosis of moderate Acute Respiratory Distress Syndrome, in substitute of the $\mathrm{PaO} 2 / \mathrm{FiO} 2$. They demonstrate that an SFR of 181 and 235 , predict a $\mathrm{PaO} 2 / \mathrm{FiO} 2$ of 200 and 300 , respectively [18].

Imaging results comprised chest radiography (CXR) abnormality and computed tomographic (CT) imaging. CXR findings were separated into three groups. Group 1 had no abnormalities, group 2 had classic changes including consolidation, pulmonary infiltrates, crazy paving pattern and ground glass opacities and in group 3 there were extensive changes of Ground glass opacities or multifocal consolidation. CT scan of the chest was done in selected patients with severe hypoxemia and to rule any other cause.

Comorbidity was defined as the presence of the conditions or history of these conditions and was extracted from electronic database. Several studies have shown that most common comorbidities including pulmonary disease, diabetes, hypertension, coronary artery disease, cerebrovascular disease, cancer and chronic renal disease were risk factors and we also evaluated these in our cohort.

The research team also collected data on degree of frailty; using the Rockwood Clinical Frailty Scale (CFS) [19] on all patients, outcomes, total length of stay and need for mechanical ventilation. CFS was documented by the clerking doctor (COVID proforma, supplementary1). Estimated CFS scores were calculated retrospectively from documentation taken no later than the first $24 \mathrm{~h}$ of admission, including clerking, past medical history, social history, previous discharge letters and any aspect of functional status.

We analysed the demographic, clinical, laboratory and imaging features of 500 patients with COVID-19 to determine potential biomarkers that may affect the prognosis of these patients. 


\section{Statistical analysis}

The baseline characteristics of all enrolled patients in survivor and non-survivor groups were summarized and compared by applying Student's $t$ test, the Chi-square test, and the Mann-Whitney $U$ test as appropriate. We did not calculate sample size prior to conducting our study. However, based on a rule of thumb, we achieved a minimum required sample size for the development of the model based on the need for 10-15 non-survived patients per risk factor [20].

Quantitative data were described by mean (standard deviation) and median (minimum-maximum). Categorical variables were summarized by frequency and percent. Bivariate analysis using Independent sample $t$-test, Mann-Whitney test as well as Pearson's Chi-square test compared different demographic and clinical parameters between survivors and non-survivors. Statistically significant and clinically relevant predictors were fitted in multivariate stepwise backward logistic regression analysis. Variables initially included were age, gender, CFS, Comorbidities > 2, NLR, CRP, creatinine, Respiratory rate, CPAP, SFR, Total Length of stay and interaction CPAP*CFS. Model selection was judged by goodness of the fit using Likelihood Ratio Test as well as pseusoR [2]. Model cross-validation was performed by randomly splitting the sample into development and test sets (ratio 3:1). The prognostic ability of the model was determined by calculating the accuracy of model's predicted probability as well as the area under the receiver operating characteristics curve (AUROC) on the test set. Statistical analysis was performed using IBM SPSS statistics program and R software packages "caTools", "Imtest", "caret", "ROCR" and "ggplot2". All statistical tests were two-sided and judged at 0.05 significance level.

\section{Results}

A total of 592 with suspected or confirmed COVID-19 were recorded during the study period, of these 500 patients with complete data set were included in the analysis. The demographic and clinical parameters of the cohort, Survivors and Non-survivors are shown in Table 1.

462 patients had positive RT-PCR nasopharyngeal swab and 38 had negative swab results. Mean age of the cohort was 69 years, 300 were Male (60\%) and 200 $(40 \%)$ were female patients. Cough and dyspnoea were the most common presentation with equal representation 294(60.2\%) followed by fever 247(49.5\%), GIT symptoms 98(19.9\%), falls 65(13\%) and confusion 47(9.4\%). Falls and confusion were common in older patients. $80(16 \%)$ patients with CFS over 4 had dementia. Majority of the cohort was Caucasian, 438(87.6\%) and age $>70$ were 291 $(58.9 \%)$. Older patients tended to present atypically, with features such as falls, confusion, decreased consciousness, poor oral intake, dizziness, general deterioration, lethargy, drowsiness and reduced mobility, Table 2 .

Of the 500 patients $193(38.6 \%)$ died. There was male preponderance among non-survivors $128(66.3 \%)$ and were much older ( 77.4 vs 64.5 years, $P<0.001)$ and presented with more comorbidities, including diabetes (65 [33.7\%] vs. 63 [20.6\%], $P=0.001$ and cardiovascular disease (95 [49.2\%] vs. 83 [27.1\%], $P<0.001)$. The proportion of deaths with $\mathrm{PaO}_{2} / \mathrm{FiO}_{2}$ less than 336 (mean) was statistically significantly $(p<0.001)$. As per ARDS criteria, nonsurvivors had lower SFR $<315, p<0.001$. Non-survivors were more tachypnoeic (respiratory rate $>24$ ) $p<0.001$.

Clinical Frailty Scale: Mean CFS was 4 , however compared to survivors of COVID- 19, non-survivors had significantly higher CFS ( 3 vs $5, p<0.001$ ). A number of laboratory parameters showed significant differences among survivors and non-survivors, Table 3. Mean CRP was significantly higher 150 vs $90, p<0.001$ in nonsurvivors, as well as neutrophil count $7.84, p<0.001$, urea 12.71, $p<0.001$ and creatinine 136, $p=0.001$.CXR abnormalities were observed more in non-survivors and supplemental oxygen requirement was higher in non-survivors $181(93.8 \%)$ as compared to survivors $165(53.9 \%)$, $p<0.001$.

There appeared to be a low incidence of superadded or co-existing bacteraemia in our patients, with most of the organisms identified from the 44 positive blood cultures being those typically associated with commensals or contamination, it was not possible to determine if any of these were nosocomial infections. The most common pathogens identified were Staphylococcus aureus, epidermidis, Staphylococcus hominis, see supplementary 2 . The COVID proforma at our hospital suggested the prescription of doxycycline and co-amoxiclav for patients who required admission, $85 \%$ of our cohort received antibiotics. However, some also received Co-trimoxazole (22\%) and Clarithromycin (17\%) as first course. Subsequently $29 \%$ required change in antibiotics for suspected secondary bacterial infection which were Tazocin (36\%), Gentamicin (16\%), Meropenem (22\%) or Vancomycin (8\%).

There were no statistical differences on length of stay, need for mechanical ventilation or symptoms between the two groups.

\section{Predictors for mortality, multivariate analysis}

Variables that were significantly associated with the outcome from univariate analysis were also entered into multivariate logistic regression models. Next, we examined various clinical parameters in the multivariate logistic regression models to identify if these were independent predictors for mortality. Age in both models was a continuous variable. 
Table 1 Demographics and baseline characteristics of the cohort

\begin{tabular}{|c|c|c|c|c|}
\hline Variables & $\begin{array}{l}\text { Total } \\
500\end{array}$ & $\begin{array}{l}\text { Survivors } \\
307\end{array}$ & $\begin{array}{l}\text { Non-Survivors } \\
193\end{array}$ & Sig* \\
\hline Male $(n, \%)$ & $300(60)$ & $172(56)$ & $128(66.3)$ & $p 0.02$ \\
\hline Female & $200(40)$ & $135(44)$ & $65(33.7)$ & \\
\hline Age in Years mean (SD) & $69.39(17.2)$ & $64.5(18.3)$ & $77.4(11.6)$ & $p<0.001$ \\
\hline Median (min-max) & $73(19-100)$ & $68(19-100)$ & $78(37-99)$ & \\
\hline IQR & $(59-83)$ & $(52-80)$ & $(70.5-87)$ & \\
\hline$<40$ & $36(7.2)$ & $35(11.4)$ & $1(0.5)$ & \\
\hline $40-70$ & $173(34.6)$ & $131(42.7)$ & $42(21.8)$ & $p<0.001$ \\
\hline $70-80$ & $132(26.4)$ & $67(21.8)$ & $60(33.7)$ & \\
\hline$>80$ & $159(31.8)$ & $74(24.1)$ & $85(44)$ & \\
\hline Ethnicity Asian $(n, \%)$ & $31(6.2 \%)$ & $24(7.8 \%)$ & $7(3.6 \%)$ & $p 0.25$ \\
\hline Black-African & $21(4.2 \%)$ & $12(3.9 \%)$ & $9(4.7 \%)$ & \\
\hline Caucasian & $438(87.6 \%)$ & $264(60 \%)$ & $174(40 \%)$ & \\
\hline Other & $10(2 \%)$ & $7(70 \%)$ & $3(30 \%)$ & \\
\hline Comorbidities, $(n, \%)<2$ & $282(56.4)$ & $203(66.1)$ & $79(40.9)$ & $p<0.001$ \\
\hline$>2$ & $218(43.6)$ & $104(33.9)$ & $114(59.1)$ & \\
\hline Diabetes Mellitus & $128(25.7)$ & $63(20.6 \%)$ & $65(33.7 \%)$ & $p 0.001$ \\
\hline Hypertension & $188(37.8)$ & $108(35.3)$ & $80(41.7)$ & $p 0.15$ \\
\hline Cardiovascular disease & $178(35.7)$ & $83(27.1)$ & $95(49.2)$ & $P<0.001$ \\
\hline Cerebrovascular disease & $44(8.8)$ & $28(9.2)$ & $16(8.3)$ & $p 0.74$ \\
\hline Respiratory disease & $148(29.7)$ & $100(32.7)$ & $48(24.9)$ & $p 0.06$ \\
\hline Other & $281(56 \%)$ & $151(49.3)$ & $132(68.4)$ & $p<0.001$ \\
\hline CFS, median (min-max) & $4(1-9)$ & $3(1-9)$ & $5(1-9)$ & $p<0.001$ \\
\hline Respiratory rate/min $>24 / \min (n, \%)$ & $271(54.2)$ & $140(45.6 \%)$ & $131(67.9)$ & $p<0.001$ \\
\hline Heart rate/min, $>100 / \min ,(n, \%)$ & $175(35)$ & $100(32.6)$ & $74(38.9)$ & $P 0.15$ \\
\hline SFR, mean (SD) & $383.5(106.8)$ & $413.0(76.6)$ & $336.8(129.1)$ & $p<0.001$ \\
\hline SFR $<235$ & $52(10.4)$ & $12(3.9)$ & $40(20.7)$ & \\
\hline $\operatorname{SFR}(235-315)(A R D S)$ & $181(10.2)$ & $21(6.8)$ & $30(15.5)$ & $p<0.001$ \\
\hline SFR $\geq 315$ (acute lung injury) & $397(79.4)$ & $274(89.3)$ & $123(63.7)$ & \\
\hline $\operatorname{Cough}(n, \%)$ & $294(60.2)$ & $186(62)$ & $108(57.4)$ & $p 0.32$ \\
\hline SOB & $294(60.2)$ & $177(59)$ & $117(62.2)$ & $p 0.45$ \\
\hline Sore throat & $32(6.5)$ & 23 (7.6) & $9(4.8)$ & $p 0.21$ \\
\hline GI & 98 (19.9) & $67(22.2)$ & $31(16.3)$ & $p 0.11$ \\
\hline Fever & $247(49.5)$ & $158(51.6)$ & $89(46.1)$ & $p 0.23$ \\
\hline Lethargy & $52(10.4)$ & $32(10.5)$ & $20(10.4)$ & $p 0.98$ \\
\hline Falls & 65 (13) & $37(12.1)$ & $28(14.5)$ & $p 0.44$ \\
\hline Myalgia & $20(4)$ & $15(4.9)$ & $5(2.6)$ & $p 0.20$ \\
\hline Confusion & $47(9.4)$ & $24(7.8)$ & 23 (11.9) & $p 0.13$ \\
\hline
\end{tabular}

CFSClinical Frailty Scale, $S F R \mathrm{SpO}_{2}$ to $\mathrm{FiO}_{2}$ ratio, $A R D S$ acute respiratory distress syndrome, $S O B$ shortness of breath, GI gastrointestinal symptoms

$\neq$ Comorbidities of interest have been selected. SFR is categorised by American European consensus

$*$ Results $\leq 0.05$ are significant, $I Q R$ interquartile range
In the stepwise logistic regression models, the following were independent risk factors for mortality in model 1, age adjusted OR 1.035 (95\% CI 1.012-1.058), NLR adjusted OR 1.021 (95\% CI 1.00-1.04), CFS adjusted OR 1.132 (95\% CI 1.13-1.53) and CRP adjusted OR 1.006 (95\% CI 1.003-1.009),
(Table 4). Again, in model 2, age and CFS score were strong risk factors. Interestingly gender did not reach the statistical significance for mortality. Similarly, creatinine, SFR and CXR abnormalities did not reach statistical significance but had a trend towards increased mortality (Table 4). 
Table 2 Most common symptoms depending on the level of clinical frailty scale

\begin{tabular}{llc}
\hline & $\begin{array}{l}\text { Clinical frailty } \\
\text { scale }<3, n(\%)\end{array}$ & $\begin{array}{c}\text { Clinical frailty } \\
\text { scale }>4, n(\%)\end{array}$ \\
\hline Cough & $156(31)$ & $137(28)$ \\
Dyspnoea & $143(29)$ & $150(30)$ \\
Fever & $133(27)$ & $113(23)$ \\
GIT & $59(12)$ & $38(7.6)$ \\
Falls & $10(2)$ & $54(11)$ \\
Confusion & $4(0.8)$ & $42(8.4)$ \\
Chest pain & $22(4.4)$ & $8(1.6)$ \\
Headache & $22(4.4)$ & $4(0.8)$ \\
Decreased consciousness & $2(0.4)$ & $10(2)$ \\
Poor oral intake & $11(2.2)$ & $6(1.2)$ \\
Dizziness & $2(0.4)$ & $6(1.2)$ \\
Sore throat & $22(4.4)$ & $9(1.8)$ \\
Lethargy & $25(5)$ & $26(5.2)$ \\
Myalgia & $20(4)$ & $2(0.4)$ \\
Reduced mobility & 0 & $12(2.4)$ \\
\hline
\end{tabular}

\section{Discussion}

This retrospective study identified several risk factors for poor outcomes in hospitalised adults with COVID-19.

The striking observation was the high mortality rate in our cohort, $38 \%$ compared to the national average of $33 \%$ [21]. One plausible explanation is that a large proportion of population in Southend are retired and older, 19\% over 65 years of age compared to national average of $17.5 \%$ as per the office of the national statistics.

The second key finding was older age with greater frailty scores. There are very few studies which evaluated clinical frailty in patients in COVID -19. Similar to our study, an Italian group assessed frailty, which demonstrated increased in-hospital mortality, ICU admissions, independent of age and sex [22]. Another study showed that CFS, but not age, remained independently associated with mortality [23]. Three other studies demonstrated higher in-patient mortality in older and frail patients [12, 24, 25]. Furthermore, a recent meta-analysis showed that each 1-point increase in CFS was associated with $12 \%$ increase in mortality in a linear fashion [26].

Frailty describes the state that results from the physiological decline resulting from natural ageing and co-morbidities, encompassing parameters such as decreasing muscle mass and strength, declining exercise tolerance and inability to provide self-care: there may also be accompanying changes in metabolism and immune system response and there is an increased vulnerability to decompensation after a stressor such as COVID. Frailty should be considered in risk assessment models in future studies and clinical trials to assess interventions and meaningful outcomes.

There is substantial literature emphasizing the importance of geriatric medicine toward frailty prevention and clinical criteria to rapidly identify those with frailty or pre-frailty $[27,28]$. Frailty, rather than simply chronological age, is considered to make patients vulnerable to decompensation and hard to recover loss of physiological reserve. This was clearly reflected in the recent COVID -19 pandemic, particularly in countries such as Italy.

In a prospective study of older patients with community acquired pneumonia, nursing home residency was an independent risk factor for viral pneumonia, which highlights the role of frailty in institutionalised populations [29] and is associated with worse outcomes in hospitalized older patients [30, 31]. The UK NICE guidelines suggest that CFS can be used as part of a holistic assessment in appropriate patients to support clinical decision-making regarding management including ceiling of care decisions. However, empirical evidence supporting the use of frailty instruments to predict treatment outcomes and triage accordingly is lacking [32].

Thirdly, our results confirmed that comorbidities, in particular cardiovascular disease and diabetes were strongly associated with negative outcomes. This is consistent with recent meta-analysis, from CDC China [33]. Similarly, another study of 5700 hospitalised patients with COVID19 in the New York City area, the most common comorbidities were hypertension (57\%), obesity (42\%), and diabetes (34\%) [34]. Other studies have reported that hypertension increases the risk odds for death in patients with COVID19 [35, 36]; however, our study did not find hypertension to be statistically significant. While hypertension does appear to be associated with more severe disease and increased mortality, there is no strong evidence to indicate increased susceptibility of patients with hypertension to COVID-19 [37]. The mechanisms of this possible relationship and their clinical relevance have been reviewed in a recent statement of the European Society of Hypertension. The putative relationship between hypertension and COVID-19 may relate to the role of ACE 2(angiotensin-converting enzyme) [37]. Diabetes, lung disease, and obesity are now well-recognised major predictors of poor clinical outcomes in many clinical scenarios. These aspects emphasize the importance of the need for multidisciplinary assessment and treatment, including cardiovascular risk evaluation and therapy, during the course of COVID-19 to reduce mortality.

Data show European mortality is generally higher in older patients compared to earlier reports from China. Age was observed as an independent predictor of mortality in our cohort, which was consistent with the large prospective UK ISARIC study of hospitalised patients [21] and Chinese data 
Table 3 Baseline clinical parameters and Laboratory studies at presentation

\begin{tabular}{|c|c|c|c|c|}
\hline Variables & Total & Survivors & Non-survivors & Sig* \\
\hline CRP mg/L, mean (SD) & $114.19(91.26)$ & $90.64(82.18)$ & $150.27(93)$ & $P<0.001$ \\
\hline Lymphocyte $10^{9} / \mathrm{L}$, mean (SD) & $1.37(4.56)$ & $1.35(3.8)$ & $1.39(5.5)$ & $P 0.93$ \\
\hline Neutrophil $10^{9} / \mathrm{L}$, mean $(\mathrm{SD})$ & $6.88(4.8)$ & $6.29(4.3)$ & $7.84(5.42)$ & $P<0.001$ \\
\hline NLR, mean (SD) & $12.56(24.5)$ & $10.62(27.86)$ & $15.65(17.72)$ & $p 0.03$ \\
\hline Urea mmol/L, mean (SD) & $9.88(8.92)$ & $8.06(7.98)$ & $12.71(9.58)$ & $P<0.001$ \\
\hline Creatinine umol/L, mean (SD) & $114.65(107.8)$ & $100.89(93.31)$ & $136.05(124.40)$ & $P 0.001$ \\
\hline Chest X-Ray, $n(\%)$ Not done & $28(5.6 \%)$ & & & \\
\hline Normal & $140(29.7)$ & $103(36)$ & 37 (19.9) & \\
\hline Moderate changes & $317(67.2)$ & $177(61.9)$ & $140(75.3)$ & $P<0.001$ \\
\hline Severe changes & $15(3.2)$ & $6(2.1)$ & $9(4.8)$ & \\
\hline CT Scan Not done, $n(\%)$ & $397(79.4 \%)$ & & & \\
\hline Covid changes & $103(20.6 \%)$ & & & \\
\hline Blood cultures, $n(\%)$ & & & & $p 0.65$ \\
\hline Gram positive & $34(6.8 \%)$ & $16(11.2)$ & $18(14.8)$ & \\
\hline Gram negative & $10(2.0 \%)$ & $6(4.2)$ & $4(3.3)$ & \\
\hline No growth & $221(83.4)$ & $121(84.6)$ & $100(82)$ & \\
\hline Not done & $235(47)$ & & & \\
\hline Oxygen requirement, $\mathrm{n}(\%)$ & $346(69.3)$ & $165(53.9)$ & $181(93.8)$ & $P<0.001$ \\
\hline CPAP & $56(11.2 \%)$ & $36(11.7)$ & $20(10.4)$ & $p 0.64$ \\
\hline Mechanical ventilation & $64(12.8 \%)$ & $36(11.7)$ & $28(14.5)$ & $p 0.37$ \\
\hline Length of stay, mean (SD) & $9.33(12.17)$ & $9.51(14.01)$ & $9.05(8.5)$ & $p 0.68$ \\
\hline Total IMV days, mean (SD) & $14.5(12.31)$ & $17.62(14)$ & $10.60(8.55)$ & $p 0.02$ \\
\hline SFR, mean (SD) & $383.65(106.81)$ & $413.08(76.68)$ & $336.82(129.19)$ & $P<0.001$ \\
\hline Baseline Fio2, mean (SD) & $0.46(4.19)$ & $0.54(5.53)$ & $0.35(0.23)$ & $p 0.62$ \\
\hline Baseline S02, mean (SD) & $91.92(8.61)$ & $93.29(8.08)$ & $89.75(9)$ & $P<0.001$ \\
\hline Respiratory rate, mean (SD) & $25.75(8.29)$ & $24.29(7.44)$ & $28.07(9.03)$ & $P<0.001$ \\
\hline
\end{tabular}

*Results $\leq 0.05$ are significant by either independent sample $t$ test, Mann-Whitney test for CFS and Chisquare test for categorical variables

$C R P C$-reactive protein, $N L R$ Neutrophil Lymphocyte ratio, CXR chest X-Ray, CXR 1 Normal, CXR 2Classic/Moderate, $C X R 3$ severe COVID changes, $C T$ computer tomography, $C P A P$ continuous positive airway pressure, IMVinvasive mechanical ventilation, $S F R \mathrm{SPO} 2$ to $\mathrm{FiO} 2$ ratio, $\mathrm{FiO} 2$ fraction of inspired oxygen, SO2 saturation of oxygen
[38-40]. In Italian studies, case fatality rates ranged from 35.5 to $52.5 \%$ in patients aged over 70 years with COVID infection [41-44]. In the USA, older patients aged $\geq 65$ years accounted for higher deaths, with the highest incidence of severe outcomes in patients aged $\geq 85$ years [45]. Why the disease is particularly dangerous in older people is not yet known and poorly understood at the molecular level. It is clear, however, that advanced age alone is by far the most significant risk factor, independent of underlying comorbidities [46, 47]. An abundance of recent data describing the pathology and molecular changes in COVID-19 patients points to both immunosenescence and inflammaging as major drivers of the high mortality rates in older patients.

In contrast to the literature, male sex was not associated with increased mortality in our study. Large studies from
China, Europe and Italy established that males were more susceptible to COVID-19-related complications, representing between 50 and $82 \%$ of the hospitalized patients with COVID -19 [5, 8, 48, 49].

Within our cohort, increased baseline CRP, creatinine and NLR were associated with poorer prognosis. The most consistent prognostic markers in COVID-19 across the different studies were elevated levels of CRP, LDH, lymphopenia and neutrophil-to-lymphocyte ratio (NLR), and these appear to stratify patients into higher risk of complications [50-52]. Intriguingly, elevated levels of C-reactive protein appear to be unique to COVID-19 patients when compared to other viral infections. Other consistently reported markers in non-survivors are increased procalcitonin (PCT) and IL-6 levels [53]. 
Table 4 Multivariate logistic regression analysis for assessing independent predictors for mortality

\begin{tabular}{|c|c|c|c|}
\hline & Sig & Unadjusted OR (95\% CI) & Adjusted OR (95\% CI) \\
\hline \multicolumn{4}{|l|}{ Model 1} \\
\hline Age & $0.002 *$ & $1.05(1.03-1.06)$ & $1.035(1.012-1.058)$ \\
\hline Neutrophil to lymphocyte ratio(NLR) & $0.024 *$ & $1.04(1.02-1.06)$ & $1.021(1.00-1.04)$ \\
\hline CFS & $<0.001 *$ & $1.45(1.30-1.62)$ & $1.132(1.13-1.53)$ \\
\hline CRP & $<0.001 *$ & $1.006(1.004-1.009)$ & $1.006(1.003-1.009)$ \\
\hline Creatinine & 0.070 & $1.004(1.001-1.008)$ & $1.002(0.999-1.005)$ \\
\hline SFR & $<0.001 *$ & $0.993(0.990-0.995)$ & $0.995(0.993-0.998)$ \\
\hline Constant & $<0.001^{*}$ & & $0.020(0-0.01)$ \\
\hline \multicolumn{4}{|l|}{ Model 2} \\
\hline Age & $<0.001 *$ & $1.05(1.03-1.06)$ & $1.038(1.013-1.063)$ \\
\hline Male gender & 0.113 & & $1.583(0.896-2.797)$ \\
\hline Neutrophil to lymphocyte ratio(NLR) & 0.050 & $1.04(1.02-1.06)$ & $1.019(0.999-1.039)$ \\
\hline CFS & $<0.001 *$ & $1.45(1.30-1.62)$ & $1.356(1.145-1.606)$ \\
\hline CRP & $<0.001 *$ & $1.006(1.004-1.009)$ & $1.006(1.002-1.009)$ \\
\hline Creatinine & 0.121 & $1.004(1.001-1.008)$ & $1.002(0.999-1.005)$ \\
\hline SFR & 0.094 & $0.993(0.990-0.995)$ & $0.997(0.994-1)$ \\
\hline$L O S$ & 0.038 & $0.997(0.979-1.015)$ & $0.971(0.946-0.998)$ \\
\hline Supp Oxygen (Yes) & $<0.001^{*}$ & $12.25(5.94-25.25)$ & $7.66(3.24-18.10)$ \\
\hline$C X R$ & 0.059 & $1.73(1.22-2.46)$ & $1.585(0.981-2.56)$ \\
\hline Constant & & & $0(0-0.01)$ \\
\hline
\end{tabular}

Model1: Variables initially included: Age, gender, CFS, Comorbidities $>2$, Neutrophil to lymphocyte ratio(NLR)

CRP, creatinine, RR, CPAP, SFR, Total LOS, interaction CPAP*CFS

Model2: Variables initially included were the same as model $1+$ Supp 02 and CXR.pseuso ${ }^{2}$ model $1=25.2 \%$, pseuso ${ }^{2}{ }_{\text {model } 2}=32.6 \%$ which mean Sup 02 as a significant predictor could explain additional $7.4 \%$ of variance in mortality outcome

Model 1 cross validation accuracy on test $\mathrm{set}=78.3 \%$, AUROC $=0.842$. Model 2 cross validation accuracy on test set $=78.1 \%$, AUROC $=0.871$

\section{Limitations}

The findings of this study are derived from hospitalised cases which might have introduced a bias in disease severity and fatality. We did not collect more details on pre-existing comorbidities and severity. The data collection is limited to what is documented in the electronic patient database whether there may be errors both with patient and clinician recall. Our single centre findings may not be generalizable. Routine tests such as LDH, Ferritin, D-Dimer and Troponin were not carried out on all patients, even with the introduction of an agreed COVID investigation panel part way through the period of interest.

\section{Conclusion}

In this large retrospective study, we found that older age, comorbidities, frailty and elevated CRP at admission were significant risk factors for poor outcomes in patients with COVID-19. Our findings add to the emerging reports quantifying the relationship between frailty and mortality in COVID-19.

Now, more than ever, a holistic approach to patients with comorbidities is required, and rapid solutions to support this must be identified and implemented with urgency. Older patients are particularly susceptible to adverse clinical outcomes in COVID-19 infection and assessment and treatment is challenging. Long-stay residential care homes and hospitals need to urgently design adequate health care plans for older patients. Our results strengthen the NICE guidance on the Clinical Frailty Scale, to assist decision-making regarding hospitalization. We suggest integrating the frailty assessment in all COVID-19 patients at hospital admission, which can help clinicians in their decision-making processes. However, shared decision-making is always warranted with respect to personal wishes and preferences of the patient. Given the economic and resource constraints, shifting hospice and palliative care resources to the community was a key message in a recent review to inform practice in the pandemic [54].

A frailty-based risk-stratification approach, rather than age may prove more valuable when considering 
interventions in patients with multiple comorbidities. The planning strategies perhaps should include awareness, tools to facilitate communication with healthcare professionals, improved access to institutional health communication and better access to local and social support activities. In addition, standardising investigations to allow early risk stratification, and inform good quality decision-making at the front door would be key to identifying the most appropriate location for, and level of care that an individual patient should receive ideally this approach should be supported by early involvement of clinicians with sufficient experience to make those decisions. In light of the patient demographics, an ideal scenario would involve geriatricians being heavily involved in planning and delivering these services.

Supplementary Information The online version contains supplementary material available at https://doi.org/10.1007/s41999-021-00508-1.

Author contributions All authors contributed to data collection, first draft GK, SG and all authors approved final version. Dr. GK had full access to all of the data and takes responsibility for the integrity of the data and accuracy of the data analysis. Analysis of the data was performed by Dr. Iman El Sayed.

Funding None received.

\section{Declarations}

Conflict of interest Dr. GK, SG, MD, VW, FH, ID and YA have nothing to disclose.

Ethical approval This study was conducted in accordance with the Institutional review board and approved by the Southend University Hospital NHS Foundation Trust and granted a waiver due to its retrospective observational design.

Competing interests All authors had no competing interests.

\section{References}

1. Who.int (2020) WHO Situation Report-163. Available at: $<$ https://www.who.int/docs/default-source/coronaviruse/situationreports/20200701-covid-19-sitrep-163.pdf?sfvrsn=c202f05b_2>. Accessed 2 July 2020.

2. Yang J, Zheng Y, Gou X et al (2020) Prevalence of comorbidities and its effects in coronavirus disease 2019 patients: a systematic review and meta-analysis. Int J Infect Dis 94:91-95

3. Grasselli G, Zangrillo A, Zanella A et al. Baseline characteristics and outcomes of 1591 patients infected with SARS- CoV-2 admitted to ICUs of the Lombardy Region, Italy. JAMA doi: https://doi.org/10.1001/jama.2020.5394. Accessed 7 Apr 2020

4. Petrilli CM, Jones SA, Yang J et al. Factors associated with hospitalization and critical illness among 4,103 patients with COVID-19 disease in New York City [e-pub ahead of print]. medRxiv. Doi: https://doi.org/10.1101/2020.04.08.20057794. Accessed 14 Apr 2020
5. Zhou F, Yu T, Du R et al (2020) Clinical course and risk factors for mortality of adult inpatients with COVID-19 in Wuhan, China: a retrospective cohort study. Lancet 395:1054-1062

6. Ortiz-Prado E, Simbaña-Rivera K, Gómez- Barreno L et al (2020) Clinical, molecular, and epidemiological characterization of the SARS-CoV-2 virus and the Coronavirus Disease 2019 (COVID-19), a comprehensive literature review. Diagn Microbiol Infect Dis 98(1):115094. https://doi.org/10.1016/j. diagmicrobio.2020.115094

7. Baud D, Qi X, Nielsen-Saines K et al (2020) Real estimates of mortality following COVID-19 infection. Lancet Infect Dis. https://doi.org/10.1016/S1473-3099(20)

8. Ruan S (2020) Likelihood of survival of coronavirus disease 2019 (comment, published online). Lancet Infect Dis. https:// doi.org/10.1016/S1473-3099(20)30257-7

9. Wu Z, McGoogan JM (2020) Characteristics of and important lessons from the coro- navirus disease 2019 (COVID-19) outbreak in China: summary of a report of 72314 cases from the Chinese Center for Disease Control and Prevention. JAMA 323(13):1239-1242. https://doi.org/10.1001/jama.2020.2648

10. Basic D, Shanley C (2015) Frailty in an older inpatient population: using the clinical frailty scale to predict patient outcomes. J Aging Health 27:670-685. https://doi.org/10.1177/08982 64314558202

11. Hewitt J, Carter B, McCarthy K, Pearce L, Law J, Wilson FV, Tay HS, McCormack C, Stechman MJ, Moug SJ et al (2019) Frailty predicts mortality in all emergency surgical admissions regardless of age. An observational study. Age Ageing 48:388-394. https:// doi.org/10.1093/ageing/afy217

12. Brill SE, Jarvis HC, Ozcan E et al (2020) COVID-19: a retrospective cohort study with focus on the over-80s and hospitalonset disease. BMC Med 18:194. https://doi.org/10.1186/ s12916-020-01665-Z

13. Hewitt J, Carter B, Vilches-Moraga A, Quinn TJ, Braude P, Verduri A et al (2020) The effect of frailty on survival in patients with COVID-19 (COPE): a multicentre, European, observational cohort study. Lancet Publ Health 5(8):e444-e451

14. Beigel J, Tomashek K, Dodd L et al (2020) Remdesivir for the treatment of Covid-19-preliminary report. N Engl J Med. https:// doi.org/10.1056/NEJMoa2007764

15. Horby PW, Lim W, Emberson J et al (2020) Effect of dexamethasone in hospitalized patients with COVID-19-preliminary report. MedRxiv 13:1

16. Kwack WG, Lee DS, Min H, Choi YY, Yun M, Kim Y, Lee SH, Song I, Park JS, Cho YJ, Jo YH, Yoon HI, Lee JH, Lee CT, Lee YJ (2018) Evaluation of the $\mathrm{SpO} 2 / \mathrm{FiO} 2$ ratio as a predictor of intensive care unit transfers in respiratory ward patients for whom the rapid response system has been activated. PLoS ONE 13(7):e0201632. https://doi.org/10.1371/journal.pone.0201632

17. Rice TW, Rice TW, Wheeler AP, Bernard GR, Hayden DL, Schoenfeld DA, Ware LB, National Institutes of Health, National Heart, Lung, and Blood Institute ARDS Network (2007) Comparison of the $\mathrm{SpO} 2 / \mathrm{FIO} 2$ ratio and the $\mathrm{PaO} 2 / \mathrm{FIO} 2$ ratio in patients with acute lung injury or ARDS. Chest 132(2):410-417

18. Bilan N, Dastranji A, Ghalehgolab BA (2015) Comparison of the spo2/fio2 ratio and the pao2/fio2 ratio in patients with acute lung injury or acute respiratory distress syndrome. J Cardiovasc Thorac Res 7(1):28-31. https://doi.org/10.15171/jcvtr.2014.06

19. Rockwood K et al (2005) A global clinical measure of fitness and frailty in elderly people. CMAJ 173:489-495. https://doi. org/10.1503/cmaj.050051

20. Field A (2013) Discovering statistics using IBM SPSS statistics. Sage

21. Docherty AB, Harrison EM, Green CA et al (2020) Features of 20133 UK patients in hospital with covid-19 using the ISARIC 
WHO clinical characterisation protocol: prospective observational cohort study. BMJ 369:m1985

22. Bellelli G, Rebora P, Valsecchi MG et al (2020) Frailty index predicts poor outcome in COVID-19 patients. Intensive Care Med 25:1-3. https://doi.org/10.1007/s00134-020-06087-2

23. De Smet R, De Smet R, Mellaerts B, Vandewinckele $\mathrm{H}$ et al (2020) Frailty and mortality in hospitalized older adults with COVID-19: retrospective observational study. J Am Med Dir Assoc. https://doi.org/10.1016/j.jamda.2020.06.008

24. Blomaard LC, der Jvan CM, van der Linden Bol JM et al (2021) Frailty is associated with in-hospital mortality in older hospitalised COVID-19 patients in the Netherlands: the COVID-OLD study. Age Ageing. https://doi.org/10.1093/ageing/afab018

25. Hagg S, Jylhävä J, Wang Y (2020) Age, frailty, and comorbidity as prognostic factors for short-term outcomes in patients with coronavirus disease 2019 in geriatric care. JAMDA 21:1555e 1559

26. Pranata R, Henrina J, Lim MA, Lawrensia S, Yonas E, Vania $\mathrm{R}$ et al (2020) Clinical frailty scale and mortality in COVID19: a systematic review and dose-response meta-analysis. Arch Gerontol Geriatr 93:104324

27. Dent E, Dent E, Morley JE, Cruz-Jentoft AJ et al (2019) Physical frailty: ICFSR international clinical practice guidelines for identification and management. J Nutr Health Aging 23(9):771-787

28. Fried LP, Tangen CM, Walston J et al (2001) Frailty in older adults: evidence for a phenotype. J Gerontol A Biol Sci Med Sci 56(3):M146-M156

29. Ma HM, Lee KP, Woo J (2013) Predictors of viral pneumonia: the need for viral testing in all patients hospitalized for nursing home acquired pneumonia. Geriatr Gerontol Int 13:949-957

30. Ellis HL, Wan B, Yeung M et al (2020) Complementing chronic frailty assessment at hospital admission with an electronic frailty index (FI-Laboratory) comprising routine blood test results. CMAJ 192(1):E3-E8

31. Muscedere J, Waters B, Varambally A et al (2017) The impact of frailty on intensive care unit outcomes: a systematic review and meta-analysis. Intensive Care Med 43(8):1105-1122

32. Hubbard RE, Maier AB, Hilmer SN et al (2020) Frailty in the face of COVID-19. Age Ageing. https://doi.org/10.1093/ageing/ afaa095

33. Liu Z, Bing X, Zhi XZ (2020) The epidemiological characteristics of an outbreak of 2019 novel coronavirus diseases (COVID19) in China Epidemiology Working Group for NCIP Epidemic Response, Chinese Center for Disease Control and Prevention. Zhonghua liu xing bing xue za zhi= Zhonghua liuxingbingxue zazhi 41(2): 145-151

34. Richardson S, Hirsch JS, Narasimhan M et al (2020) Northwell COVID-19 research consortium. Presenting characteristics, comorbidities, and outcomes among 5700 patients hospitalized With COVID-19 in the New York City area. JAMA. https://doi. org/10.1001/jama.2020.6775

35. Zhou F, Yu T, Du R et al (2020) Clinical course and risk factors for mortality of adult inpatients with COVID-19 in Wuhan, China: a retrospective cohort study. Lancet 395(10229):1054-1062

36. Guzik T, Mohiddin S, Dimarco A et al (2020) COVID-19 and the cardiovascular system: implications for risk assessment, diagnosis, and treatment options. Cardiovasc Res 30:cvaa106. https://doi.org/10.1093/cvr/cvaa106

37. Kreutz R, Algharably EAE, Azizi M et al (2020) Hypertension, the renin-angiotensin system, and the risk of lower respiratory tract infections and lung injury: implications for COVID-19. Cardiovasc Res 15:1

38. Guan WJ, Ni ZY, Hu Y et al (2020) Clinical characteristics of coronavirus disease 2019 in China. China medical treatment expert group for covid-19. N Engl J Med 382(18):1708-1720
39. Du RH, Liang LR, Yang CQ et al (2020) Predictors of mortality for patients with COVID-19 pneumonia caused by SARSCoV-2: a prospective cohort study. Eur Respir J 55:2000524. https://doi.org/10.1183/13993003.00524-2020

40. Li LQ, Huang T, Wang YQ et al (2019) Novel coronavirus patients' clinical characteristics, discharge rate and fatality rate of meta-analysis. J Med Virol. https://doi.org/10.1002/jmv. 25757

41. Colaneri M, Sacchi P, Zuccaro V et al (2020) Clinical characteristics of coronavirus disease (COVID-19) early findings from a teaching hospital in Pavia, North Italy, 21 to 28 February 2020. Euro Surveill 25(16): 1

42. Onder G, Rezza G, Brusaferro S (2020) Case-fatality rate and characteristics of patients dying in relation to COVID-19 in Italy. JAMA. https://doi.org/10.1001/jama.2020.4683

43. Verity R, Kell O, DorigattiI C et al (2020) Estimates of these verity of corona virus disease2019: amodel- based analysis. Lancet Infect Dis. 30:1

44. Sorbello M, El-Boghdadly K, GiacintoI D et al (2020) The Italian COVID-19 outbreak: experiences and recommendationsfromclinicalpractice. Anaesthesia 75(6):724-732

45. CDC COVID-19 Response Team (2020) Severe outcomes among patients with coronavirus disease 2019 (COVID-19): United States, February 12-March 16, 2020. MMWR Morb Mortal Wkly Rep 69(12):343-346

46. Williamson E, Walker AJ, Bhaskaran KJ et al (2020) Open SAFELY: factors associated with COVID-19-related hospital death in the linked electronic health records of 17 million adult NHS patients. MedRxiv. https://doi.org/10.1101/2020.05.06. 20092999

47. Santesmasses D, Castro JP, Zenin AA et al (2020) COVID-19 is an emergent disease of aging. MedRxiv. https://doi.org/10. $1101 / 2020.04 .15 .20060095$

48. Grasselli G, Zangrillo A, Zanella A et al (2020) Baseline characteristics and outcomes of 1591 patients infected with SARSCoV-2 admitted to ICUs of the lombardy region, Italy. JAMA 323(16):1574-1581. https://doi.org/10.1001/jama.2020.5394

49. Gebhard C, Regitz-Zagrosek V, Hannelore K et al (2020) Impact of sex and gender on COVID-19 outcomes in Europe. Biol Sex Differ 11:29. https://doi.org/10.1186/s13293-020-00304-9

50. Fei J, Fu L, Li Y et al (2020) Reduction of lymphocyte at early stage elevates severity and death risk of COVID-19 patients: a hospital-based case-cohort study. MedRxiv. https://doi.org/10. 1101/2020.04.02.20050955

51. Wynants L, Van Calster B, Bonten MMJ et al (2020) Prediction models for diagnosis and prognosis of covid-19 infection: systematic review and critical appraisal. BMJ 369:m1328

52. Zhao X, Zhang B, Li P et al (2020) Incidence, clinical characteristics and prognostic factor of patients with COVID-19: a systematic review and meta-analysis. MedRxiv. https://doi.org/ $10.1101 / 2020.03 .17 .20037572$

53. Huang C, Wang Y, Li X et al (2020) Clinical features of patients infected with 2019 novel coronavirus in Wuhan, China. Lancet 395:497-506

54. Etkind S, Bone A, Lovell $\mathrm{N}$ et al (2020) The role and response of palliative care and hospice services in epidemics and pandemics: a rapid review to inform practice during the COVID-19 pandemic. J Pain Symptom Manag. https://doi.org/10.1016/j. jpainsymman.2020.03.029

Publisher's Note Springer Nature remains neutral with regard to jurisdictional claims in published maps and institutional affiliations. 